\title{
Meta-analysis
}

\section{Mortality in psoriasis and psoriatic arthritis: systematic review and meta-analysis}

\author{
Karen Fabiola Velásquez-Hernández ${ }^{1}$, Maria Luisa Peralta-Pedrero ${ }^{1}$, \\ Miriam de Jesús Velásquez-Hernández², Alan Isaac Valderrama-Treviño ${ }^{3}$, \\ Martha Alejandra Morales-Sánchez ${ }^{1 *}$
}

\author{
${ }^{1}$ Education and Research Unit, Dermatological Center Dr. Ladislao de la Pascua, National Autonomous University of \\ Mexico, Mexico \\ ${ }^{2}$ Institute of Physical and Theoretical Chemistry, Graz University of Technology, Graz, Austria \\ ${ }^{3}$ Department of Surgery, Central North Hospital PEMEX, Mexico City, Mexico
}

Received: 19 July 2021

Revised: 01 August 2021

Accepted: 03 August 2021

*Correspondence:

Dr. Martha Alejandra Morales-Sánchez,

E-mail: marthamoralessanchez@gmail.com

Copyright: (c) the author(s), publisher and licensee Medip Academy. This is an open-access article distributed under the terms of the Creative Commons Attribution Non-Commercial License, which permits unrestricted non-commercial use, distribution, and reproduction in any medium, provided the original work is properly cited.

\begin{abstract}
We had found contradictory results that have been reported in recent publications regarding the mortality risk of patients with psoriasis (Pso) and psoriatic arthritis (PsA). These patients have aggregated risk behaviors, which directly impacted their morbidity/mortality. We included 15 studies, with a total population of that reported mortality risk in Pso and PsA patients. We calculated crude mortality rate (CMR) of each one and pooled CMR by group and 95\% confidence intervals (CI). The pooled CMR for Pso was 14/1000 (95\% CI: 6-21\%), 12/1000 (95\% CI: 10-15\%) in mild, 19/1000 (95\% CI: 15-23\%) in severe and 12/1000 was observed (95\% CI: 10-14\%) in PsA. Mortality was relatively higher in PsA patients when compared with Pso, with a RR of 1.03 (95\% CI: 1.01-1.06, p<0.01). Pso was associated with increased mortality when compared to the general population. Mild Pso and PsA have the same increased mortality, then again as the severity of Pso increased, so does its mortality. The final comparative mortality between patients with PsA and those with Pso was around 3\%.
\end{abstract}

Keywords: Psoriasis, Arthritis psoriatic, Mortality, Crude mortality rate

\section{INTRODUCTION}

Pso is a common, chronic, recurrent, inflammatory disease of the skin mediated by TH17 lymphocytes response, it affects $2-4 \%$ of the general adult population, its prevalence varies according to geography from $0.9 \%$ in USA to $8.5 \%$ in Norway and $2 \%$ of the dermatological consultation in Mexico. ${ }^{1-4}$ Pso patients developed erythematous scaly plaques and can affect nails and joints (PsA) in more than $25 \%$ of Pso patients. ${ }^{5}$ PsA is a chronic inflammatory arthritis with an estimated incidence rate of up to 6.6 per 100,000 per year. ${ }^{6}$ Both Pso and PsA have a negative physical, emotional and psychosocial impact. ${ }^{7-15}$ Proliferation and differentiation of keratinocytes are dysregulated in Pso, with an important involvement of interleukin 17 (IL17), IL23 and tumor necrosis factor (TNF). These ILs induce a proinflammatory state, insulin resistance, endothelial dysfunction and cardiovascular disease, thus explaining the increased incidence of comorbidities in Pso and PsA. However, there are other risk factors such as family history, pharmacological adverse effects as well as alcohol and tobacco consumption. ${ }^{16-20}$ Treatment depends on the severity of 
the disease, which includes a combination of topical and/or systemic agents (e.g. phototherapy, drug modifying antirheumatic drugs and biological agents). Moderate cases, topical resistant patients and cases with nail and/or articulation involvement require a more aggressive approach. ${ }^{2,21}$ Some authors have identified an increase in psoriasis mortality rate, which could be associated with the presence of comorbidities such as cardiovascular disease, neoplasms and pulmonary disease, among others. Another group has described an increased mortality risk in patients with severe psoriasis that could be associated with both co-morbidities and short and long term effects of the systemic treatment. ${ }^{2,10,21-26}$ Although it is well known that the therapy of PsA and severe psoriasis is similar, in PsA patients, the premature mortality risk is different from Pso patients. ${ }^{27}$ Recently, Dhana et al published a metaanalysis that reported an increased mortality risk in psoriasis patients, likewise Wong et al and Ali et al reported a similar increase in PsA, conversely Shbeeb et al and Wilson et al obtained different results. ${ }^{27-34}$ Unfortunately, Dhana et al systematic review did not include PsA patients, consequently we considered that it was necessary to do an update to compare the mortality between psoriatic arthropathy and Pso. Until now, mortality in Pso and PsA continued to be an enigma and it was still controversial the association between severity, co-morbidities and therapeutics. The aim of this study was to determine the risk of mortality in patients with Pso and PsA in relation to general population. We also analyzed and described the main characteristics of the cohorts that were included in this systematic review.

\section{METHODS}

We performed a systematic review according to the preferred reporting items for systematic reviews and meta-analyses statement (PRISMA) and meta-analyses of observational studies in epidemiology (MOOSE) for systematic reviews and meta-analysis. ${ }^{35,36}$
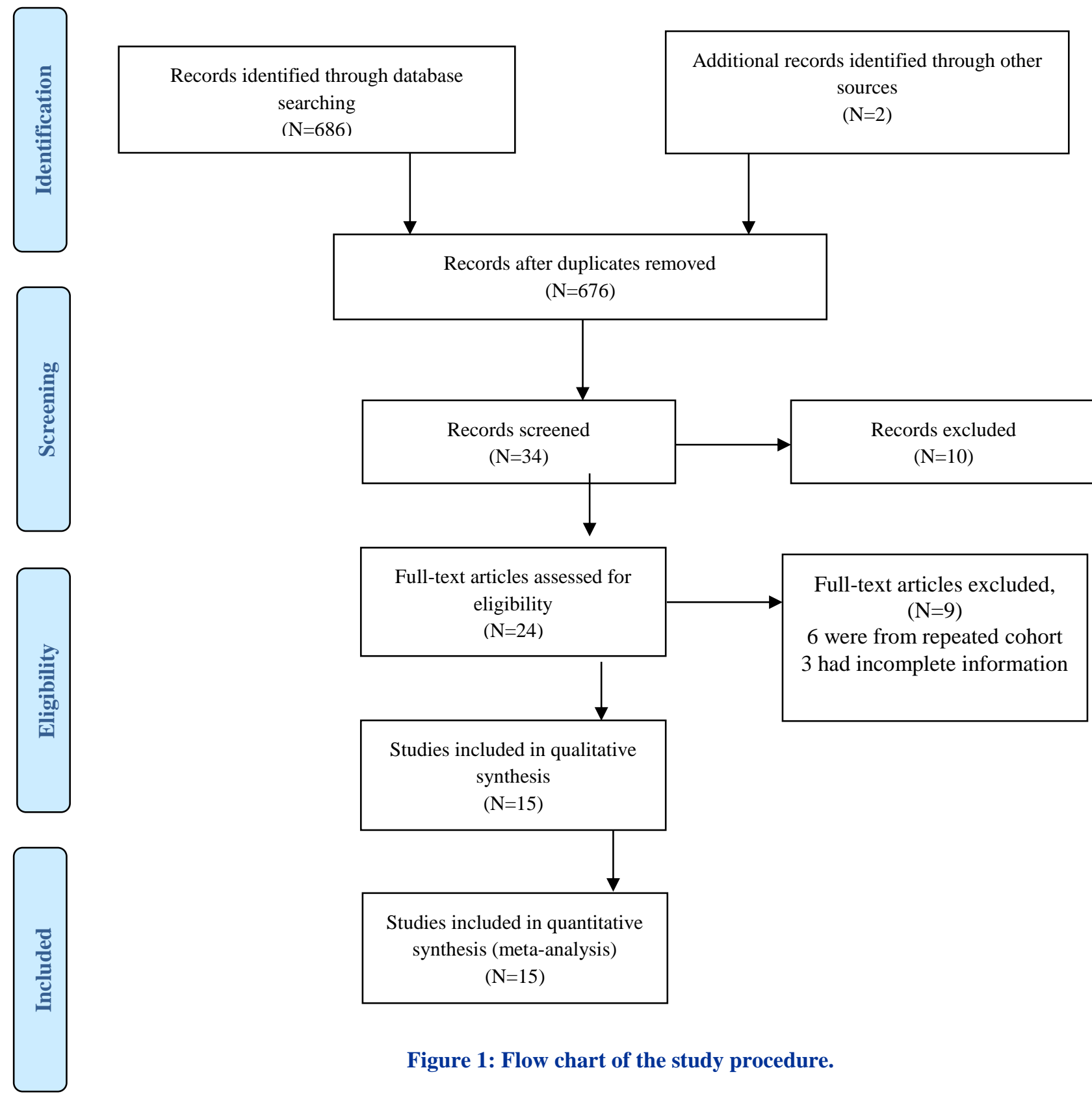

Full-text articles excluded,

6 were from repeated cohort

3 had incomplete information

Figure 1: Flow chart of the study procedure. 


\section{Data source and search}

On 23 May 2019 the electronic search was carried out in PubMed, Ovid, Web of Science, Virtual Health Library (VHL) and Cinni articles databases without date restrictions. We used the following search terms: psoriasis and mortality and cohort or prospective studies or retrospective studies. We also searched the references of the included articles. Two authors independently participated in the literature search, study selection, data extraction and quality assessment (Figure 1). Any disagreements were solved by consensus of two researchers and the intervention of a third researcher in case of doubt. Details of the protocol for this systematic review were registered on PROSPERO and can be accessed at https://www.crd.york.ac.uk/prospero/ (CRD42019123496).

\section{Inclusion criteria}

We selected cohort studies that were written in English or Spanish, whose primary outcome was mortality in adults with Pso and PsA that reported cumulative person-years and/or average follow up. If the data was available, we performed subgroups by severity of Pso and PsA.

\section{Exclusion criteria}

We excluded review articles, commentaries and editorials. Original articles that only reported a specific cause of death, had incomplete data about death or only evaluated co-morbidity and not mortality were also excluded.

\section{Data extraction and assessment of study quality}

We extracted information using tables that contained complete information from each study. We assessed study quality using a modified Newcastle-Ottawa scale 37 and classified them by their risk of bias: low (7 points), medium (5 to 6 points), or high ( $\leq 4$ points) as well as with Joanna Bridges institute (JBI) critical appraisal checklist for cohort studies. ${ }^{38}$ Study quality was determined with GRADEpro. ${ }^{39}$

\section{Statistical analysis}

For each study, CMR was calculated such as metaanalysis of Manouchehrinia and cols, pooled CMR (pCMR) in Pso and sub groups pCMR including mild, severe Pso and PsA. ${ }^{40}$ We used random effects models for analysis and calculated RR with $95 \%$ CI to be the measure of association when comparing mortality in Pso versud PsA. We chose a random effects model because of potential between-study heterogeneity, which was measured with I2. In case of a high heterogeneity, subset analysis was repeated multiple times, with removal of one or more studies each time to investigate its source. A funnel plot was used as a visual tool for assessment of publication bias. Beggs and Egger's regression test was used for the investigation of small study bias. All statistical analyses were performed with Stata V.11 (StataCorp, Stata statistical software). Statistical tests with a $p<0.05$ were considered statistically significant.

\section{RESULTS}

We found 686 articles, after reading the title and abstract, we evaluated 34 full texts including 12 Pso and 6 PsA articles. Because some articles reported cohorts from the same database, we reviewed the studies and chose the one with the most complete information or with the longest study period, in order to avoid overlapping the sample. Table 1 summarizes the main characteristics of the included articles. Studies were conducted in 7 countries: Taiwan, Argentina, Finland, Canada, United States of America, Denmark and United Kingdom. Study duration varied from 10 to 35 years.

\section{CMR in Pso}

The global CMR included data from 6 papers. These studies comprised data from 434,579 patients. A total of 32,934 deaths occurred during follow up time. The pooled CMR was 14/1000 (95\% CI: 6-21) with an $\mathrm{I}^{2}=99.9 \%, \mathrm{p}<0.01$, this global pooled result did not show publication bias by Begg's and Egger's test (Figure 2).

\section{Mild, severe Pso and arthritis psoriatic pooled CMR}

These included data from 13 papers. Among six studies with mild psoriasis, the pCMR was 12/1000 (95\% CI: $10-$ $\left.15 \%, \mathrm{I}^{2} 99.84 \%, \mathrm{p}<0.01\right)$. Eight studies for severe Pso, the pCMR was 19/1000 (95\% CI: $15-23 \%, \mathrm{I}^{2} 99.42 \%$, $\mathrm{p}<0.01)$. Six studies were included in PsA group, pCMR was 12/1000 (95\% CI: $\left.10-14 \%, \mathrm{I}^{2} 96.64 \%, \mathrm{p} \leq 0.01\right)$. Pooled CMR was 15/1000 (95\% CI: $13-17 \%$, I² $99.7 \%$, $\mathrm{p} \leq 0.01$ ), this result did not show publication bias by Begg and Eger test (Figure 3).

\section{Pso versus PsA}

We made a comparison of patients with Pso vwesus PsA. Among 4 studies of 319,085 patients with Pso and 36,890 with PsA, the results were 25,572 and 2,489 demises respectively. The pooled RR in Psa group was $1.03(95 \%$ CI: $\left.1.01-1.06 \%, \mathrm{I}^{2} 98.4 \%, \mathrm{p}<0.01\right)$ and it did not have publication bias (Figure 4).

\section{Sensitivity analyses, publication bias and study quality}

We performed sensitivity analyses, influence analyses and risk bias assessment. Studies that did not modify the results were excluded. Funnel plot, Begg and Egger's test showed no evidence of publication bias $(\mathrm{p}<0.05)$. The evidence review team conducted a series of systematic literature analysis following the methods of the Cochrane Collaboration and GRADE evidence profiles for each outcome. This particular study included observational 
studies so it was classified as low quality evidence. For the first 2 outcomes (CMR) we used a narrative description form, as it was calculated using only one group, there was no factor that increased the quality due to the design of the study (Table 2). The last outcome was also classified as low quality and degraded after we made an indirect comparison and observed some of the included articles compared the studied group versus healthy patients (Table 2). The risk of bias was evaluated with Newcastle-Ottawa scale and JBI critical appraisal checklist for cohort studies. The inconsistency found in a small number of studies was not considered serious due to the large sample size. All calculated sample sizes (TOI) were smaller than the total number of patients included.

Table 1: Main characteristics of studies.

\begin{tabular}{|c|c|c|c|c|c|c|c|}
\hline $\begin{array}{l}\text { Author, } \\
\text { year, } \\
\text { country }\end{array}$ & Settings & $\begin{array}{l}\text { Assessment } \\
\text { of } \\
\text { mortality }\end{array}$ & $\begin{array}{l}\text { Number of } \\
\text { psoriasis } \\
\text { patients }\end{array}$ & $\begin{array}{l}\text { Severity or } \\
\text { diagnosis }\end{array}$ & $\begin{array}{l}\text { Study } \\
\text { period }\end{array}$ & Years & Conclusions \\
\hline \multicolumn{8}{|c|}{ United Kingdom } \\
\hline $\begin{array}{l}\text { Gelfand et al } \\
2007\end{array}$ & GPRD & $\begin{array}{l}\text { Registratios } \\
\text { codes }\end{array}$ & $\begin{array}{l}\text { Mild } 133 \\
568, \\
\text { controls: } \\
56035, \\
\text { severe: } \\
\text { 3951, } \\
\text { controls: } 15 \\
075\end{array}$ & $\begin{array}{l}\text { Severe: patient } \\
\text { with a history } \\
\text { of systemic } \\
\text { therapy } \\
\text { mild without } \\
\text { this }\end{array}$ & $\begin{array}{l}1987- \\
2002\end{array}$ & $>18$ & $\begin{array}{l}\text { The results of this } \\
\text { study demonstrate } \\
\text { that patients with } \\
\text { severe psoriasis } \\
\text { have a } 50 \% \\
\text { increased risk of } \\
\text { mortality }\end{array}$ \\
\hline $\begin{array}{l}\text { Ogdie et al } \\
2017\end{array}$ & THIN & $\begin{array}{l}\text { Code } \\
\text { noting } \\
\text { death or } \\
\text { transfer due } \\
\text { to death }\end{array}$ & PsA: 8706 & $\begin{array}{l}\text { Single } \\
\text { diagnosis code } \\
\text { (positive } \\
\text { predictive } \\
\text { value } 85 \% \text { ) }\end{array}$ & $\begin{array}{l}1994- \\
2010\end{array}$ & $18-89$ & $\begin{array}{l}\text { Overall mortality } \\
\text { and cause-specific } \\
\text { mortality risk were } \\
\text { not elevated among } \\
\text { patients with PsA } \\
\text { except for suicide } \\
\text { deaths }\end{array}$ \\
\hline $\begin{array}{l}\text { Ogdie et al } \\
2014\end{array}$ & THIN & $\begin{array}{l}\text { Code } \\
\text { noting } \\
\text { death or } \\
\text { transfer due } \\
\text { to death }\end{array}$ & $\begin{array}{l}\text { PsA: } 8,706, \\
\text { AR:41,752, } \\
\text { Psoriasis: } \\
\text { 138,424 y } \\
\text { Controles: } \\
82,258\end{array}$ & READ codes & $\begin{array}{l}1994- \\
2010\end{array}$ & $18-89$ & $\begin{array}{l}\text { Patients with RA } \\
\text { and psoriasis had a } \\
\text { high mortality } \\
\text { compared to the } \\
\text { general population. } \\
\text { However, patients } \\
\text { with PsA did not } \\
\text { have a significantly } \\
\text { elevated risk of } \\
\text { mortality. }\end{array}$ \\
\hline $\begin{array}{l}\text { Megan et al } \\
2018\end{array}$ & THIN & NE & $\begin{array}{l}8760 \text { adults } \\
\text { with } \\
\text { psoriasis } \\
\text { and } 87,600 \\
\text { adults } \\
\text { without } \\
\text { psoriasis }\end{array}$ & $\begin{array}{l}\text { CDC y } \\
\text { National } \\
\text { psoriasis } \\
\text { foundation } \\
\text { severidad: BSA }\end{array}$ & $\mathrm{NE}$ & Adults & $\begin{array}{l}\text { Patients with } \\
\text { psoriasis affecting } \\
>10 \% \text { BSA have an } \\
\text { increased risk of } \\
\text { death compared to } \\
\text { the general } \\
\text { population, patients } \\
\text { with psoriasis and a } \\
\text { BSA > 10\% should } \\
\text { be subject to } \\
\text { preventive health } \\
\text { interventions. }\end{array}$ \\
\hline $\begin{array}{l}\text { Abuabara et } \\
\text { al } 2010\end{array}$ & GPRD & $\begin{array}{l}\text { Code } \\
\text { noting } \\
\text { death }\end{array}$ & $\begin{array}{l}\text { Severe } \\
\text { psoriasis } \\
3603 \text { and } \\
\text { controls } \\
14,330\end{array}$ & $\begin{array}{l}\text { ICD } 10, \\
\text { definition of } \\
\text { severity } \\
\text { according to } \\
\text { therapeutics }\end{array}$ & $\begin{array}{l}1987- \\
2002\end{array}$ & $>18$ & $\begin{array}{l}\text { Severe psoriasis is } \\
\text { associated with an } \\
\text { increased risk of } \\
\text { death. Due to } \\
\text { cardiovascular, } \\
\text { pulmonar and } \\
\text { neoplasic causes . }\end{array}$ \\
\hline
\end{tabular}




\begin{tabular}{|c|c|c|c|c|c|c|c|}
\hline $\begin{array}{l}\text { Author, } \\
\text { year, } \\
\text { country }\end{array}$ & Settings & $\begin{array}{l}\text { Assessment } \\
\text { of } \\
\text { mortality }\end{array}$ & $\begin{array}{l}\text { Number of } \\
\text { psoriasis } \\
\text { patients }\end{array}$ & $\begin{array}{l}\text { Severity or } \\
\text { diagnosis }\end{array}$ & $\begin{array}{l}\text { Study } \\
\text { period }\end{array}$ & Years & Conclusions \\
\hline $\begin{array}{l}\text { Buckley et al } \\
2010\end{array}$ & $\begin{array}{l}\text { Hospital } \\
\text { Base }\end{array}$ & $\begin{array}{l}\text { Registry of } \\
\text { deaths } \\
\text { (National } \\
\text { health } \\
\text { service) }\end{array}$ & PsA 453 & $\begin{array}{l}\text { Criteria for } \\
\text { PsA to Moll } \\
\text { and Wright }\end{array}$ & $\begin{array}{l}1985- \\
2007\end{array}$ & NE & $\begin{array}{l}\text { There is no } \\
\text { significant increase } \\
\text { in the risk of death } \\
\text { in patients with } \\
\text { psoriatic arthritis }\end{array}$ \\
\hline $\begin{array}{l}\text { Springate et } \\
\text { al } 2017\end{array}$ & $\begin{array}{l}\text { Clinical } \\
\text { practice } \\
\text { research } \\
\text { datalink } \\
\text { (CPRD) }\end{array}$ & NE & $\begin{array}{l}104,441 \\
\text { with } \\
\text { psoriasis } \\
\text { and } \\
508,457 \text { in } \\
\text { the control } \\
\text { group. }\end{array}$ & READ code & $\begin{array}{l}1 \text { January } \\
1999 \text { to } \\
31 \\
\text { December } \\
2013 .\end{array}$ & $\begin{array}{l}0 \text { to } \\
\text { over } \\
80\end{array}$ & $\begin{array}{l}\text { Prevalence } \\
\text { increased from } \\
2.3 \% \text { (1999) to } \\
2.8 \% \text { (2013), not at } \\
\text { the expense of } \\
\text { incidence, which is } \\
\text { explained by the } \\
\text { fact that mortality } \\
\text { in patients with } \\
\text { psoriasis has } \\
\text { decreased in UK. } \\
\text { However, early } \\
\text { mortality in these } \\
\text { patients remains } \\
\text { high compared to } \\
\text { the general } \\
\text { population (HR } \\
1.53 \text { in patients } \\
\text { aged 0-19 years } \\
\text { with psoriasis). }\end{array}$ \\
\hline $\begin{array}{l}\text { Svedbom et } \\
\text { al } 2015\end{array}$ & $\begin{array}{l}\text { VEGA and } \\
\text { SHCR }\end{array}$ & CDR & $\begin{array}{l}\text { cohort } 1 \\
(136,409 \\
\text { individuals } \\
\text { in control } \\
\text { group, } \\
34,355 \\
\text { cases with } \\
\text { mild } \\
\text { psoriasis). } \\
\text { cohort } 2 \\
\text { (18,366 } \\
\text { patients in } \\
\text { the control } \\
\text { group, } 4,719 \\
\text { patients } \\
\text { with severe } \\
\text { psoriasis). }\end{array}$ & ICD 10 & $\begin{array}{l}\text { SHCR: } 1 \\
\text { January } \\
2001 \text { to } \\
31 \\
\text { December } \\
2010 \\
\text { VEGA: } 1 \\
\text { January } \\
2005 \text { to } \\
31 \text { March } \\
2011\end{array}$ & NE & $\begin{array}{l}\text { In patients with } \\
\text { mild or severe } \\
\text { psoriasis, the } \\
\text { greatest } \\
\text { associations were } \\
\text { observed due } \\
\text { demise caused by } \\
\text { kidney and liver } \\
\text { disease, however in } \\
\text { general there was } \\
\text { an increase in } \\
\text { mortality in } \\
\text { patients with } \\
\text { psoriasis from all } \\
\text { causes. }\end{array}$ \\
\hline \multicolumn{8}{|l|}{ Denmark } \\
\hline $\begin{array}{l}\text { Salahadeen et } \\
\text { al } 2015\end{array}$ & $\begin{array}{l}\text { The central } \\
\text { population } \\
\text { registry, the } \\
\text { National } \\
\text { patient } \\
\text { registry e } \\
\text { información } \\
\text { de la } \\
\text { prescripción }\end{array}$ & $\begin{array}{l}\text { The } \\
\text { National } \\
\text { causes of } \\
\text { death } \\
\text { registry }\end{array}$ & $\begin{array}{l}\text { Psoriasis: } \\
5,458,627, \\
\text { mild: } \\
94,069 \text { and } \\
\text { severe: } \\
28,253\end{array}$ & ICD 10, ICD 8 & $\begin{array}{l}1997- \\
2011\end{array}$ & $>18$ & $\begin{array}{l}\text { Higher rates in all } \\
\text { the specific causes } \\
\text { of death, mainly } \\
\text { cardiovascular (for } \\
\text { every } 1000 \text { patients } \\
\text { year } 3.6 \text { mild and } \\
5.2 \text { severe), } \\
\text { malignant diseases } \\
\text { (rate } 3.9 \text { mild and } \\
5.4 \text { severe) and } \\
\text { gastrointestinal } \\
\text { (rate } 0.9 \text { mild and } \\
1.8 \text { severe). }\end{array}$ \\
\hline
\end{tabular}




\begin{tabular}{|c|c|c|c|c|c|c|c|}
\hline $\begin{array}{l}\text { Author, } \\
\text { year, } \\
\text { country }\end{array}$ & Settings & $\begin{array}{l}\text { Assessment } \\
\text { of } \\
\text { mortality }\end{array}$ & $\begin{array}{l}\text { Number of } \\
\text { psoriasis } \\
\text { patients }\end{array}$ & $\begin{array}{l}\text { Severity or } \\
\text { diagnosis }\end{array}$ & $\begin{array}{l}\text { Study } \\
\text { period }\end{array}$ & Years & Conclusions \\
\hline $\begin{array}{l}\text { Skov et al } \\
2019\end{array}$ & NPR & $\begin{array}{l}\text { Civil } \\
\text { registration } \\
\text { system }\end{array}$ & $\begin{array}{l}\text { Psoriasis: } 12 \\
160 \text { Control: } \\
23936 \\
\text { PsA: } 9817 \\
\text { Control: } \\
19398\end{array}$ & ICD 10 & $\begin{array}{l}1998- \\
2014\end{array}$ & NE & $\begin{array}{l}\text { Patients with } \\
\text { psoriasis have an } \\
\text { increased risk of } \\
\text { mortality (HR } \\
\text { 1.74), but not in } \\
\text { patients with PsA } \\
\text { (HR 1.06). }\end{array}$ \\
\hline \multicolumn{8}{|l|}{ Argentina } \\
\hline $\begin{array}{l}\text { Masson et al } \\
2017\end{array}$ & $\begin{array}{l}\text { Hospital } \\
\text { database }\end{array}$ & $\begin{array}{l}\text { In-hospital } \\
\text { or out-of } \\
\text { hospital } \\
\text { death }\end{array}$ & $\begin{array}{l}1,481 \\
\text { patients } \\
\text { with } \\
\text { psoriasis } \\
\text { and } 1,500 \\
\text { without it }\end{array}$ & $\begin{array}{l}\text { Medical } \\
\text { records }\end{array}$ & $\begin{array}{l}1 \text { January } \\
2010 \text { to } \\
\text { 30 June } \\
2015\end{array}$ & $\begin{array}{l}2010- \\
2015\end{array}$ & $\begin{array}{l}\text { In the univariate } \\
\text { analysis, patients } \\
\text { with psoriasis } \\
\text { showed } 58 \% \text { more } \\
\text { mortality than the } \\
\text { non-exposed group. } \\
\text { in the multivariate } \\
\text { analysis, psoriasis } \\
\text { was associated with } \\
\text { higher mortality } \\
\text { compared to the } \\
\text { control group (HR } \\
\text { 1.48) }\end{array}$ \\
\hline \multicolumn{8}{|c|}{ United States of America } \\
\hline $\begin{array}{l}\text { Stern et al } \\
2011\end{array}$ & $\begin{array}{l}\text { University } \\
\text { clinic } \\
\text { centers }\end{array}$ & NDI & 1380 & ICD 9 & $\begin{array}{l}1977- \\
2005\end{array}$ & NE & $\begin{array}{l}\text { Patients with severe } \\
\text { and very extensive } \\
\text { psoriasis showed an } \\
\text { increased risk of } \\
\text { mortality from all } \\
\text { causes compared to } \\
\text { the general } \\
\text { population and } \\
\text { people with less } \\
\text { extensive psoriasis. } \\
\text { These increases } \\
\text { were not significant } \\
\text { due to } \\
\text { cardiovascular } \\
\text { disease (HR 1.42) }\end{array}$ \\
\hline \multicolumn{8}{|l|}{ Canada } \\
\hline Ali et al 2007 & $\begin{array}{l}\text { Hospital } \\
\text { database } \\
\text { PsA clinic }\end{array}$ & $\begin{array}{l}\text { Linkage } \\
\text { with the } \\
\text { provincial } \\
\text { cancer } \\
\text { registry, } \\
\text { telephone } \\
\text { interviews, } \\
\text { newspaper. } \\
\text { Death } \\
\text { certificates. }\end{array}$ & PsA 680 & NE & $\begin{array}{l}1978- \\
2004\end{array}$ & $\begin{array}{l}15.5- \\
87.5\end{array}$ & $\begin{array}{l}\text { The risk of } \\
\text { mortality in } \\
\text { patients with PsA is } \\
\text { decreasing over } \\
\text { time }\end{array}$ \\
\hline \multicolumn{8}{|l|}{ Taiwan } \\
\hline Dai et al 2018 & NHIRD & $\begin{array}{l}\text { Withdrawal } \\
\text { of } \\
\text { insurance } \\
\text { patients }\end{array}$ & $\begin{array}{l}\text { Psoriasis } \\
106,701, \\
\text { PsA } 8795\end{array}$ & $\begin{array}{l}\text { Diagnosis: ICD } \\
9 \\
\text { Severity } \\
\text { according to } \\
\text { the therapeutic }\end{array}$ & $\begin{array}{l}2002- \\
2012\end{array}$ & $\begin{array}{l}\text { Older } \\
\text { and } \\
\text { less } \\
\text { than } \\
18\end{array}$ & $\begin{array}{l}\text { Patients with } \\
\text { psoriasis have a } \\
\text { higher risk of } \\
\text { mortality compared } \\
\text { to controls, while } \\
\text { the severity of } \\
\text { psoriasis and PsA }\end{array}$ \\
\hline
\end{tabular}




\begin{tabular}{|c|c|c|c|c|c|c|c|}
\hline $\begin{array}{l}\text { Author, } \\
\text { year, } \\
\text { country }\end{array}$ & Settings & $\begin{array}{l}\text { Assessment } \\
\text { of } \\
\text { mortality }\end{array}$ & $\begin{array}{l}\text { Number of } \\
\text { psoriasis } \\
\text { patients }\end{array}$ & $\begin{array}{l}\text { Severity or } \\
\text { diagnosis }\end{array}$ & $\begin{array}{l}\text { Study } \\
\text { period }\end{array}$ & Years & Conclusions \\
\hline & & & & & & & $\begin{array}{l}\text { had no impact on } \\
\text { mortality risk }\end{array}$ \\
\hline $\begin{array}{l}\text { Lee et al } \\
2017\end{array}$ & $\begin{array}{l}\text { National } \\
\text { health } \\
\text { insurance } \\
\text { database }\end{array}$ & $\begin{array}{l}\text { National } \\
\text { death } \\
\text { registry of } \\
\text { Taiwan }\end{array}$ & $\begin{array}{l}\text { Psoriasis } \\
80167 \\
\text { PsA } 9572\end{array}$ & $\begin{array}{l}\text { ICD } 9 \\
\text { Severe: if } \\
\text { patients } \\
\text { received } \\
\text { systemic } \\
\text { therapeutic } \\
\text { agents and mild } \\
\text { if they did not } \\
\text { receive these }\end{array}$ & $\begin{array}{l}2001- \\
2012\end{array}$ & $\geq 18$ & $\begin{array}{l}\text { Patients with severe } \\
\text { psoriasis, early- } \\
\text { onset psoriasis and } \\
\text { PsA had higher } \\
\text { mortality risks from } \\
\text { various causes. }\end{array}$ \\
\hline
\end{tabular}

GPRD (general practitioners participating in the general practice research database), THIN (the health improvement network), NE (not specified),CDC (center for disease control and prevention), BSA (body surface área), NPR (Danish National patient registry ), NHIRD (National health insurance research database), CRD (the causes of death register), NDI (National death index), RA (rheumatoid arthritis), ICD (International classification of diseases ), SHCR (Skåne health care register), HR (hazzard ratio).

Table 2: Grade quality assessment.

\section{Certainty assessment}
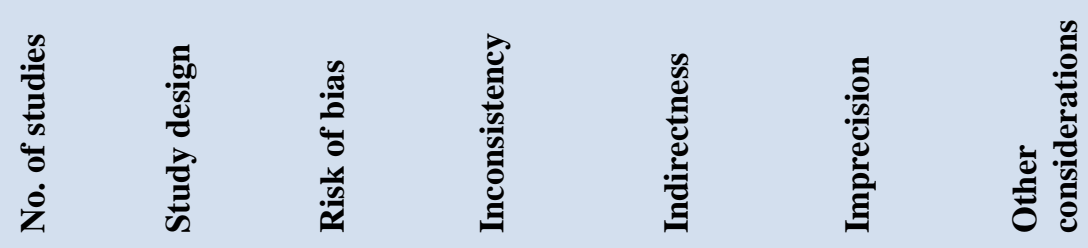

Impact Certainty Importance

CMR in Pso

\begin{tabular}{|c|c|c|c|c|c|c|c|c|}
\hline 6 & $\begin{array}{l}\text { Observat } \\
\text { ional } \\
\text { studies }\end{array}$ & $\begin{array}{l}\text { Not } \\
\text { serious }\end{array}$ & $\begin{array}{l}\text { Not } \\
\text { serious }\end{array}$ & $\begin{array}{l}\text { Not } \\
\text { serious }\end{array}$ & $\begin{array}{l}\text { Not } \\
\text { serious }\end{array}$ & None & $\underset{\text { low }}{\oplus \oplus \bigcirc \bigcirc{ }^{a}}$ & Important \\
\hline \multicolumn{9}{|c|}{ CMR subgroup mild, severe and PsA } \\
\hline 12 & $\begin{array}{l}\text { Observat } \\
\text { ional } \\
\text { studies }\end{array}$ & $\begin{array}{l}\text { Not } \\
\text { serious }\end{array}$ & $\begin{array}{l}\text { Not } \\
\text { serious }\end{array}$ & $\begin{array}{l}\text { Not } \\
\text { serious }\end{array}$ & $\begin{array}{l}\text { Not } \\
\text { serious }\end{array}$ & None & $\begin{array}{l}\oplus \oplus \bigcirc \bigcirc \\
\text { low }\end{array}$ & Important \\
\hline
\end{tabular}

Table 3: Grade summary of findings.

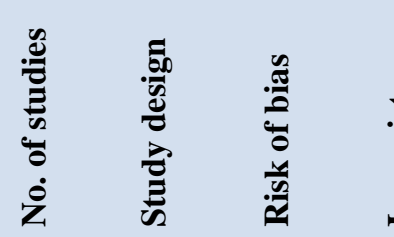

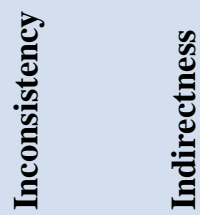
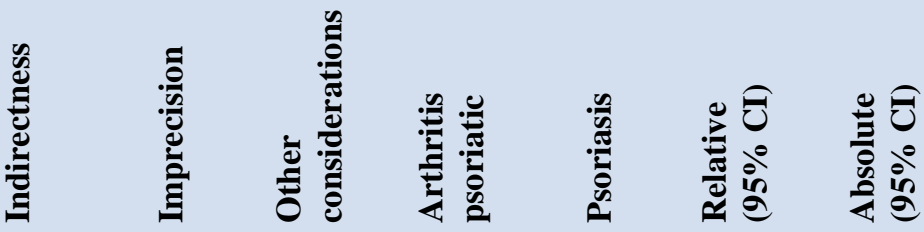

\section{Pso versus PsA}

\begin{tabular}{|c|c|c|c|c|c|c|c|c|c|c|c|c|}
\hline 4 & $\begin{array}{l}\text { Observ } \\
\text { ational } \\
\text { studies }\end{array}$ & $\begin{array}{l}\text { Not } \\
\text { seri } \\
\text { ous }\end{array}$ & $\begin{array}{l}\text { Not } \\
\text { serious }\end{array}$ & Serious ${ }^{b}$ & $\begin{array}{l}\text { Not } \\
\text { serious }\end{array}$ & None & $\begin{array}{l}2489 / 3 \\
6890 \\
(6.7 \%)\end{array}$ & $\begin{array}{l}25572 / \\
319085 \\
(8.0 \%)\end{array}$ & $\begin{array}{l}\text { RR } \\
1.03 \\
(1.01 \\
\text { to } \\
1.06)\end{array}$ & $\begin{array}{l}2 \text { more } \\
\text { per } 1.000 \\
\text { (from } 1 \\
\text { more to } 5 \\
\text { more) }\end{array}$ & $\begin{array}{l}\bigoplus \\
\bigcirc \\
\bigcirc \\
\bigcirc \\
\text { ver } \\
\text { y } \\
\text { low }\end{array}$ & $\begin{array}{l}\text { Impor } \\
\text { tant }\end{array}$ \\
\hline
\end{tabular}

CI: confidence interval; RR: risk ratio; a: certainty is low because these are observational studies that don't include considerations for an increase in risk factors and so it does not adapt to our study; b: we realized comparison of patient with Pso and PsA and some included studies did each comparison versus healthy people. 
Table 4: Limitations reported by study.

\begin{tabular}{|c|c|}
\hline Authors/cohort & Reported limitations \\
\hline Gelfand et al 2007 & Risk of misclassification \\
\hline Ogdie et al 2017 & $\begin{array}{l}\text { Lack of death certificate information and the inferential nature of } \\
\text { assigning cause of death and they lack information on disease } \\
\text { activity }\end{array}$ \\
\hline Ogdie et al 2014 & $\begin{array}{l}\text { It was not possible to prove mortality according to severity, } \\
\text { misclassification of diagnoses and lack of information regarding } \\
\text { the use of DMARDs }\end{array}$ \\
\hline Megan et al 2017 & $\begin{array}{l}\text { Future research is needed to better elucidate the specific causes of } \\
\text { mortality in patients with extensive psoriasis and to determine the } \\
\text { effects of the treatment of psoriasis on the risk of mortality }\end{array}$ \\
\hline Abuabara et al 2010 & $\begin{array}{l}\text { Classification of severity according to the treatment, possibility of } \\
\text { attending only patients who request attention (which could include } \\
\text { only patients with serious injuries) }\end{array}$ \\
\hline Springate et al 2016 & $\begin{array}{l}\text { Psoriasis cases were identified from general practice electronic } \\
\text { health records using relevant diagnostic code lists and so may not } \\
\text { necessarily have been verified by dermatologists, this study } \\
\text { includes only those patients who present in general practice and } \\
\text { thereby receive a physician diagnosis of psoriasis, but this would } \\
\text { also be true in other patient populations }\end{array}$ \\
\hline Svedbom et al 2015 & $\begin{array}{l}\text { Retrospective study. Some individuals in the database probably } \\
\text { had psoriasis and were not diagnosed }\end{array}$ \\
\hline Salahadeen et al 2014 & $\begin{array}{l}\text { Caucasian population. Reported death causes by a doctor were } \\
\text { taken . Patients with psoriasis without treatment or treated only } \\
\text { with topical steroids could have been omited, which could lead to } \\
\text { an underestimation of the death rate, whereas identifying patients } \\
\text { with hospital management could lead to an increase in } \\
\text { comorbidities }\end{array}$ \\
\hline Skov et al 2018 & $\begin{array}{l}\text { Retrospective study, probable diagnostic error, hospital-based } \\
\text { study which could have biased the result, information regarding } \\
\text { the therapist was not included, the cause of death was assigned by } \\
\text { a single investigator }\end{array}$ \\
\hline Masson et al 2017 & $\begin{array}{l}\text { The use of a secondary database may cause information bias, no } \\
\text { clinimetric tests were performed for an adequate classification, no } \\
\text { specific mortality was evaluated, including hospital population } \\
\text { could increase comorbidities at the time of diagnosis }\end{array}$ \\
\hline Stern et al 2011 & Excludes pregnant patients \\
\hline Poikolainen et al 1999 & $\begin{array}{l}\text { There is no information regarding the treatment with methotrexate, } \\
\text { which could contribute to hepatopathy }\end{array}$ \\
\hline Dai et al 2018 & $\begin{array}{l}\text { Probable misclassification of severity given that the registry does } \\
\text { not include clinimetric evaluations (use of treatment patterns as a } \\
\text { marker of severity), the causes of death could not be identified, } \\
\text { death was taken at the time of insurance withdrawal, however, it } \\
\text { could be due to renounce citizenship }\end{array}$ \\
\hline Buckley et al 2015 & $\begin{array}{l}\text { Probably the results may not be extendable to the entire } \\
\text { population, given that only white population was included }\end{array}$ \\
\hline Ali et al 2007 & $\mathrm{NE}$ \\
\hline Lee et al 2017 & $\begin{array}{l}\text { The absence of clinical assessments limited our ability to classify } \\
\text { the severity of psoriasis using the physician global assessment and } \\
\text { psoriasis assessment severity index, excess causes of death due to } \\
\text { adverse effects of anti-psoriatic therapies, unhealthy lifestyles, } \\
\text { comorbidity, and other factors could not be controlled for in the } \\
\text { SMR analyses }\end{array}$ \\
\hline
\end{tabular}




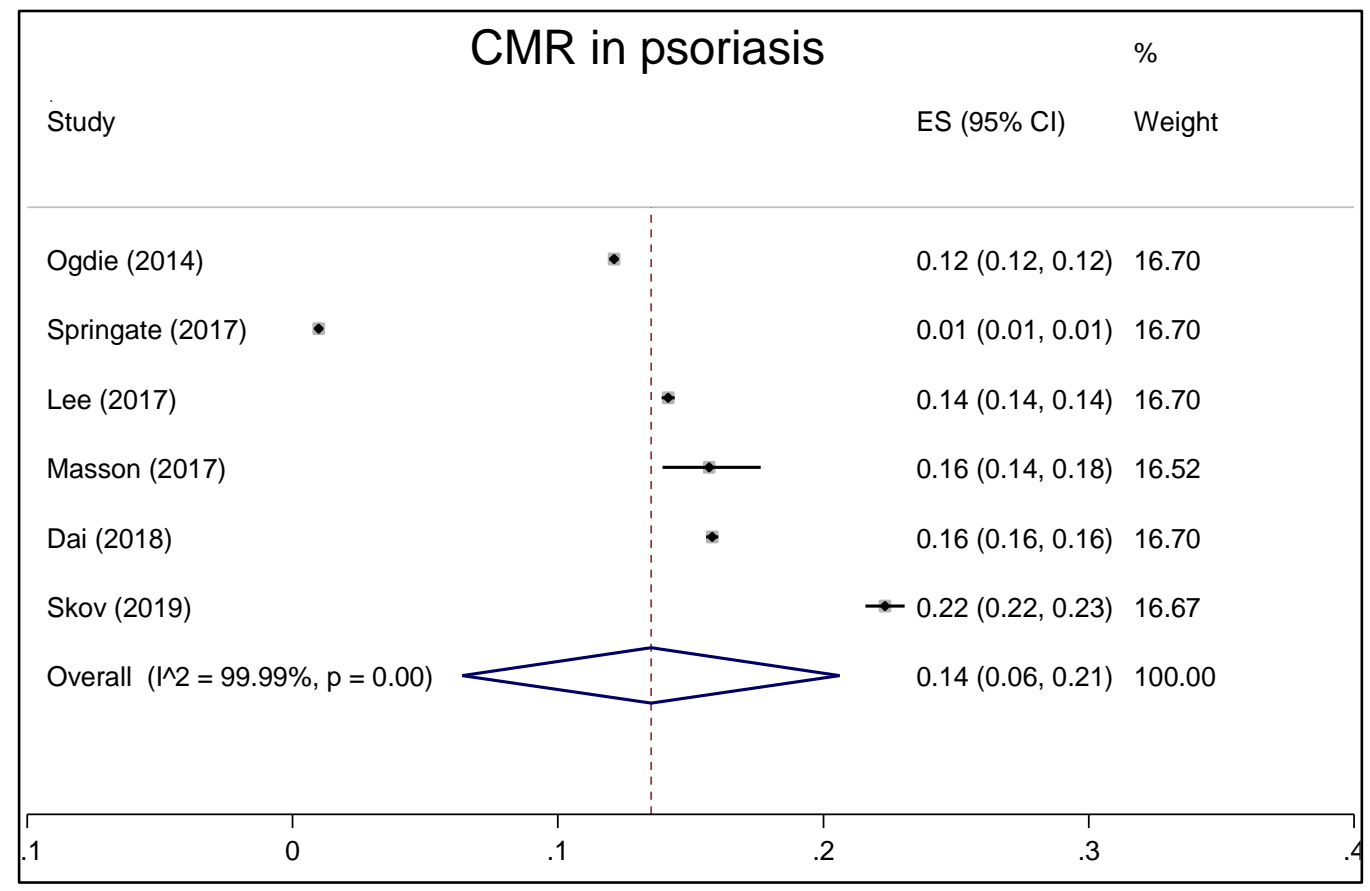

Figure 2: Forest plot of CMR in psoriasis patients.

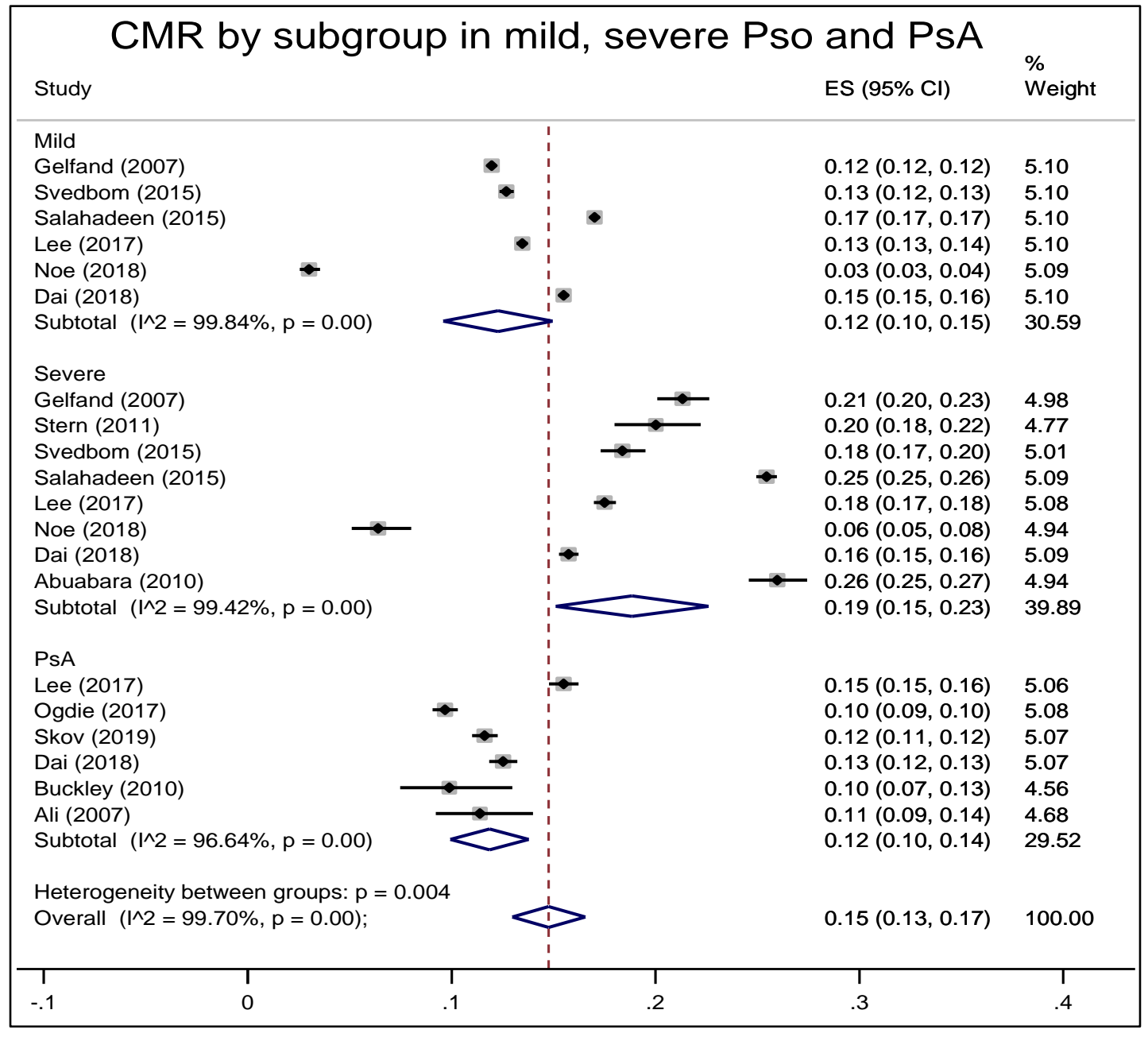

Figure 3: Forest plot of CMR in mild, severe Pso and PsA patients. 


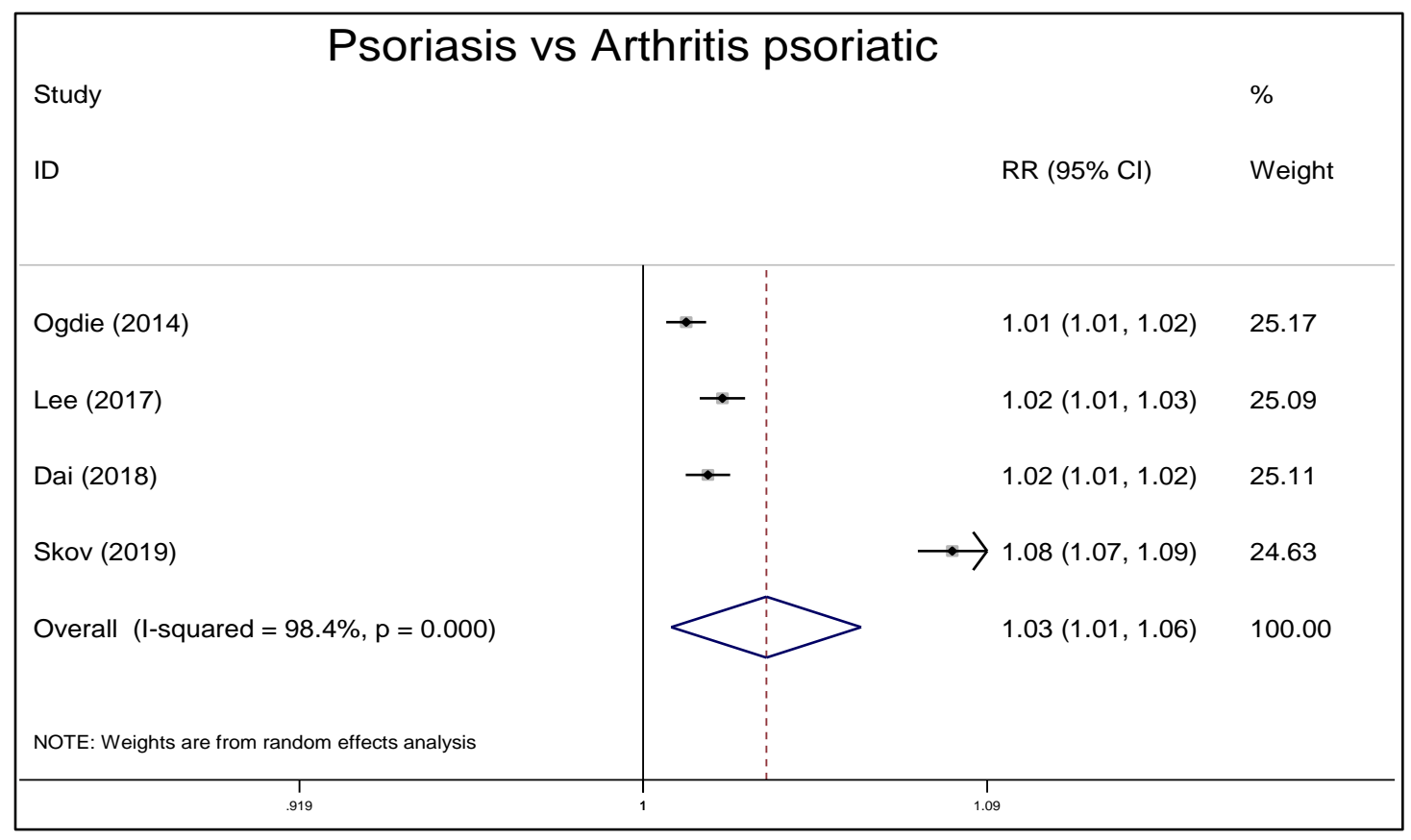

Figure 4: Forest plot of mortality in Pso versus PsA patients.

\section{DISCUSSION}

According to our meta-analysis, the CMR for Pso was 14 deaths per 1,000 population (95\% CI: 6-21). Our study was the first to summarize the mortality data using CMR and compare it to data from general population. Comparing our data with the ten leading causes of death in high-income and upper-middle-income countries in 2016, Pso CMR was ten times higher than the CMR of ischemic heart disease ( 1.45 deaths per 1000 population), which was the leading cause of death among these countries. ${ }^{41}$ When we compared our data with the general CMR in 2017 of Denmark (8.2 deaths per 1000 people), United Kingdom (9.2 deaths per 1000 people), Argentina (7.56 deaths per 1000 people), Canada (7.5 deaths per 1000 people), United States of America (8.5 deaths per 1000 people) and China (7.11 deaths per 1000 people), Pso mortality was also higher with 14 deaths per 1000 individuals. ${ }^{42}$ These countries were selected to make the comparison because most of the cohorts included in this meta-analysis contain data from national registries of those populations, but compared to all countries we observed a CMR similar to the countries with the highest CMR such as Bulgaria, Croatia, Hungary (15.5, 13, 13.5 respectively). ${ }^{43}$ In these countries there was a demographic aging, one of the central characteristics in the population of the most developed countries. ${ }^{44,45}$ When we analyzed data by severity of the disease, mild Pso had a CMR similar to PsA, while in severe Pso the CMR was higher than the global, 19 deaths per 1000 people. Compared with the CMR per country, mortality in psoriasis patients was also higher than mortality in general population. The similarity of mild Pso and PsA
CMR's observed in our study suggested that mortality increase was not associated with the systemic therapy administered for PsA (drug modifying antirheumatic drugs DMARDs and biologic therapy). Pearce et al, Gelfand et al and Gulliver 20 have discussed an association (risk or protective) of pharmacological therapy and mortality in psoriasis, but we considered it was a spurious association as we have added factors to the equation including the burden of other diseases frequently found in this population such as cardiovascular, infectious and neoplastic conditions..$^{20,22,46}$ Mortality risk in PsA patients is a controversial issue despite the results of three cohort studies from Taiwan, United Kingdom and Denmark. ${ }^{27,34,47}$ The first showed an increase of mortality risk (HR: 1.52 95\% CI: 1.39-1.66) while the others reported no association with mortality (HR: 0.94 95\% CI: 0.80-1.10, HR: 1.06, $\mathrm{p}=0.19$ respectively). In our meta-analysis, we compared PsA patients versus $\mathrm{PsO}$ and observed a marginally increase in mortality risk of 3\% (RR: 1.03 95\% CI: 1.01-1.06).

The limitations of our investigation were the studies were heterogeneous in several aspects such as definition of severe Pso (not clinimetrically evaluated); chronic tobacco use, obesity and sedentary lifestyle may contribute to the maintenance of a proinflammatory state and were not taken into account in some articles. Table 4 contained the limitation reported in each study.

\section{CONCLUSION}

With these findings, we can conclude that patients with psoriasis have an increased risk of mortality in comparison to general population as well as patients with 
psoriatic arthritis have a slight increased risk when compared to patients with Pso. Further studies are needed to assess the causality of metabolic disease on mortality risk in Pso and PsA. Our study has several strengths. We perform a calculation of the CMR with the intention to unify values and to be able to compare the general mortality rate with the one associated with Pso and PsA. We included patients with PsA, no similar studies have been found to this date

Our study suggested that regardless of the severity; the patients with Pso and PsA should receive appropriate screening and preventive intervention. We consider that it is important to perform a clinimetric evaluation to accurately determine the severity of the disease in order to improve the evaluation of each patient. The mortality in psoriasis and PsA has not been modified over the years, despite the pharmacological improvements.

\section{ACKNOWLEDGEMENTS}

Authors would like to thank the Education and Research Unit of Dermatological Center Dr. Ladislao de la Pascua for support and dedication.

\section{Funding: No funding sources}

Conflict of interest: None declared

Ethical approval: Not required

\section{REFERENCES}

1. Han JH, Lee JH, Do HK, Seo H, Bang CH, Park $\mathrm{YM}$, et al. Epidemiology and medication trends in patients with psoriasis: a nationwide populationbased cohort study from Korea. Acta Derm Venereol. 2018;98(4):396-400.

2. Sui LM, Yi-Chun Y, Ting CY, Lai M. All-cause and cause-specific mortality in patients with psoriasis in taiwan: a nationwide population-based study. J Invest Dermatol. 2017;137(7):1468-73.

3. Mehta NN, Azfar RS, Shin DB, Neimann AL, Troxel AB, Gelfand JM. Patients with severe psoriasis are at increased risk of cardiovascular mortality: cohort study using the general practice research database. Eur Heart J. 2010;31(8):1000-6.

4. Chanussot C, Arenas R. Psoriasis, a descriptive study of comorbidities in 114 patients. Dermatologia CMQ. 2015;13(1):20-3.

5. Griffiths CEM, Barker JNWN. Pathogenesis and clinical features of psoriasis. Lancet. 2007;370(9583):263-71.

6. Buckley C, Cavill C, Taylor G, Kay H, Waldron N, Korendowych E, et al. Mortality in psoriatic arthritis-a single-center study from the UK. J Rheumatol. 2010;37(10):2141-4.

7. Ogdie A, Maliha S, Shin D, Love TJ, Baker J, Jiang $\mathrm{Y}$, et al. Cause-specific mortality in patients with psoriatic arthritis and rheumatoid arthritis. Rheumatology (Oxford). 2017;56(6):907-11.
8. Puig L. Cardiometabolic comorbidities in psoriasis and psoriatic arthritis. Int J Mol Sci. 2018;19(1):58.

9. Parisi R, Symmons DPM, Griffiths CEM, Ashcroft $\mathrm{DM}$, Identification and management of psoriasis and associated comorbidity (IMPACT) project team. Global epidemiology of psoriasis: a systematic review of incidence and prevalence. J Invest Dermatol. 2013;133(2):377-85.

10. Griffiths CEM, Walt JMVD, Ashcroft DM, Flohr C, Naldi L, Nijsten $T$, et al. The global state of psoriasis disease epidemiology: a workshop report. Br J Dermatol. 2017;177(1):4-7.

11. Gupta R, Debbaneh MG, Liao W. Genetic epidemiology of psoriasis. Curr Dermatol Rep. 2014;3(1):61-78.

12. Egeberg A, Skov L, Gislason GH, Thyssen JP, Mallbris L. Incidence and prevalence of psoriasis in Denmark. Acta Derm Venereol. 2017;97(7):808-12.

13. Duarte GV, Silva LP, Oliveira MDFPD. Epidemiology and treatment of psoriasis: a Brazilian perspective. Psoriasis (Auckl). 2015;5:55-64.

14. Langley R, Krueger GG, Griffiths C. Psoriasis: epidemiology, clinical features, and quality of life. Ann Rheum Dis. 2005;64(2):18-23.

15. Hae JS, Chul JP, Tae YK, Choe YB, Lee S, Kim NI, et al. The clinical profile of patients with psoriasis in korea: a nationwide cross-sectional study (EPIPSODE). Ann Dermatol. 2017;29(4):462-70.

16. Noe MH, Shin DB, Wan MT, Gelfand JM. Objective measures of psoriasis severity predict mortality: a prospective population- based cohort study. J Invest Dermatol. 2018;138(1):228-30.

17. Salahadeen E, Torp-Pedersen C, Gislason G, Hansen PR, Ahlehoff O. Nationwide populationbased study of cause-specific death rates in patients with psoriasis. J Eur Acad Dermatol Venereol. 2015;29(5):1002-5.

18. Boehncke WH, Boehncke S. Cardiovascular mortality in psoriasis and psoriatic arthritis: epidemiology, pathomechanisms, therapeutic implications, and perspectives. Curr Rheumatol Rep. 2012;14(4):343-8.

19. Horreau C, Pouplard C, Brenaut E, Barnetche T, Misery L, Cribier B, et al. Cardiovascular morbidity and mortality in psoriasis and psoriatic arthritis: a systematic literature review. J Eur Acad Dermatol Venereol. 2013;27(3):12-29.

20. Gulliver W. Long-term prognosis in patients with psoriasis. Br Assoc Dermatol. 2008;159(2):2-9.

21. Stern RS, Huibregtse A. Very severe psoriasis is associated with increased noncardiovascular mortality but not with increased cardiovascular risk. J Invest Dermatol. 2011;131(5):1159-66.

22. Pearce DJ, Higgins KB, Stealey KH, Balkrishnan R, Crane MM, Camacho F, et al. Adverse events from systemic therapies for psoriasis are common in clinical practice. J Dermatolog Treat. 2006;17(5):288-93.

23. Masson W, Rossi E, Galimbe ML, Krauss J, Estrada JN, Galimberti R, et al. Mortality in patients with 
psoriasis. Analysis of a retrospective cohort. Medicina Clinica Barcelona. 2017;148(11):483-8.

24. Abuabara K, Azfar RS, Shin DB, Neimann AL, Troxel AB, Gelfand JM. Cause-specific mortality in patients with severe psoriasis: a population-based cohort study in the UK. $\mathrm{Br} J$ Dermatol. 2010;163(3):586-92.

25. Svedbom A, Dalen J, Mamolo C, Cappelleri JC, Mallbris L, Petersson IF, et al. Increased causespecific mortality in patients with mild and severe psoriasis: a population-based Swedish register study. Acta Derm Venereol. 2015;95(7):809-15.

26. Armstrong AW, Schupp C, Bebo B. Psoriasis comorbidities: results from the National psoriasis foundation surveys 2003 to 2011. Dermatology. 2012;225(2):121-6.

27. Ogdie A, Haynes K, Troxel AB, Love TJ, Hennessy $\mathrm{S}$, Choi $\mathrm{H}$, et al. The risk of mortality in patients with psoriatic arthritis, rheumatoid arthritis, and psoriasis: a longitudinal cohort study. Ann Rheum Dis. 2014;73(1):149-53.

28. Dhana A, Yen H, Yen H, Cho E. All-cause and cause-specific mortality in psoriasis: a systematic review and meta-analysis. J Am Acad Dermatol. 2019;80(5):1332-43.

29. Wong K, Gladman DD, Husted J, Long JA, Farewell VT. Mortality studies in psoriatic arthritis. Arthritis Rheum. 1997;40(10):1868-72.

30. Yaser A, Brian DMT, Schentag CT. Improved survival in psoriatic arthritis with calendar time. Arthritis Rheum. 2007;56(8):2708-14.

31. Shbeeb M, Uramoto K, Gibson L, O'Fallon WM, Gabriel SE. The epidemiology of psoriatic arthritis in Olmsted County, Minnesota, USA, 1982-1991. J Rheumatol. 2000;27(5):1247-50.

32. Wilson FC, Icen M, Crowson CS, McEvoy MT, Gabriel SE, Kremers HM. Time trends in epidemiology and characteristics of psoriatic arthritis over 3 decades: a population-based study. J Rheumatol. 2009;36(2):361-7.

33. Springate D, Parisi R, Kontopantelis E, Reeves D, Griffiths CEM, Ashcroft DM. Incidence, prevalence and mortality of patients with psoriasis: a U.K. population-based cohort study. $\mathrm{Br} \mathrm{J}$ Dermatol. 2017;176(3):650-8.

34. Ying-Xiu D, Ming-Chun H, Hsiao-Yun H, Chang Y, Chen T, Li C, et al. The risk of mortality among psoriatic patients with varying severity: a nationwide population-based cohort study in Taiwan. Int J Environ Res Public Health. 2018;15(12):2622.
35. Moher D, Shamseer L, Clarke M, Ghersi D, Liberati A, Petticrew M, et al. Preferred reporting items for systematic review and meta-analysis protocols (PRISMA-P) 2015 statement. Syst Rev. 2015;4(1):1.

36. Stroup DF, Berlin JA, Morton SC, Olkin I, Williamson GD, Rennie D, et al. Meta-analysis of observational studies in epidemiology. JAMA. 2000;283(15):2008-12.

37. Wells G, Shea B, Connel D, Peterson J, Welch V, Losos M, et al. The Newcastle-Ottawa scale (NOS) for assessing the quailty of nonrandomised studies in meta-analyses. Ottawa Hosp. 2009.

38. Moola S, Munn Z, Tufanaru C. Chapter 7: Systematic reviews of etiology and risk. Joanna Briggs Instit Rev Manual. 2017.

39. GRADEpro Guideline Development Tool. Fact sheet: McMaster University, 2015. Available at: https://gradepro.org/cite/. Accessed on 2 July 2021.

40. Manouchehrinia A, Tanasescu R, Tench CR, Constantinescu CS. Mortality in multiple sclerosis: meta-analysis of standardised mortality ratios. J Neurol Neurosurg Psychiatry. 2016;87(3):324-31.

41. WHO. Fact sheet: The top 10 causes of death, 2016. Available at: https://www.who.int/news-room/factsheets/detail/the-top-10-causes-of-death. Accessed on 2 July 2021.

42. Knoema Corporation. Fact sheet: Population estimates and projections, 2017. Available at: https://knoema.com/WBPEP2018Oct/populationestimates-andprojections? country $=1000530 \&$ series $=1000140$. Accessed on 2 July 2021.

43. The world bank. Death rate, crude (per 1,000 people), 2017. Available at: https://data.worldbank.org/indicator/SP.DYN.CDRT .IN?contextual=aggregate. Accessed on 2 July 2021.

44. Alcañiz M. Demographic changes in global society. Pap Poblac. 2008;14(57).

45. Bloom DE, Luca DL. The global demography of aging: facts, explanations, future. IZA. 2016.

46. Gelfand JM, Troxel AB, Lewis JD. The risk of mortality in patients with psoriasis. Archiv Dermatol. 2007;143(12):1493-9.

47. Skov L, Thomsen SF, Kristensen LE. Causespecific mortality in patients with psoriasis and psoriatic arthritis. Br J Dermatol. 2019;180:100-7.

Cite this article as: Velásquez-Hernández KF, Peralta-Pedrero ML, Velásquez-Hernández MJ, Valderrama-Treviño AI, Morales-Sánchez MA. Mortality in psoriasis and psoriatic arthritis: systematic review and meta-analysis. Int J Res Med Sci 2021;9:2804-15. 